\title{
Criterios tecnológico-ambientales bajo un enfoque sistémico: transferencia de tecnología química
}

\section{Technological Criteria Technology-Environmental under a Systemic Approach: Chemistry Technology Transfer}

\author{
Durán-García Martín Enrique \\ Departamento de Tecnología Industrial \\ División de Ciencias Administrativas y Tecnológicas \\ Universidad Simón Bolívar \\ Correos:martinenriqueduran@gmail.comymartinduran@usb.ve
}

Información del artículo: recibido: marzo de 2013, reevaluado: abril de 2012, octubre de 2012 y marzo de 2013, aceptado: mayo de 2013

\section{Resumen}

En la actualidad la transferencia de la tecnología química es un proceso que contribuye con las políticas tecnológicas de un país, una industria o una organización química en general. Este proceso, requiere la aplicación de criterios claros que permitan el adecuado desarrollo de las complejas interrelaciones existentes al momento de transferir una tecnología química. Un grupo de los criterios que están presentes son los relacionados con los tecnológicos y ambientales, los cuales definen intrínsicamente a la tecnología y su impacto con el medio ambiente. Por lo tanto, la transferencia de la tecnología química requiere criterios tecnológico-ambientales que definen, en conjunto con los demás criterios, un proceso adecuado para la selección, adquisición e incorporación de una tecnología bajo un enfoque holístico, de manera que proporcione soluciones factibles a la industria química en pro de sus objetivos. Entonces el criterio se convierte en un referente para evaluar un proceso de transferencia tecnológica adecuado. Se realiza un análisis teórico de los criterios tecnológicos y ambientales, logrando proponer treinta y seis (36) criterios tecnológico-ambientales que se interrelacionan bajo un enfoque sistémico en el proceso de transferencia de la tecnología química, a partir de una primera corrida del ciclo metodológico basado principalmente en el método de investigación-acción. Se espera en futuras investigaciones realizar un refinamiento de los criterios a partir de la formulación y validación de sus métricas de manera que se realicen los ajustes necesarios que optimicen el proceso de transferencia de la tecnología química.

\section{Descriptores:}

- transferencia tecnológica

- criterios ambientales

- tecnología química

- enfoque holístico

- método investigación-acción 


\begin{abstract}
Currently the transfer of chemical technology is a process that contributes to the technology policy of a country, an industry or an organization in general chemistry. This process requires the application of clear criteria for the proper development of the complex interrelations in the transfer of chemical technology. A group of criteria that are present, are those related to environmental technology which intrinsically define the technology and its impact to the environment. Therefore, the transfer of chemical technology requires technological-environmental criteria defining, in conjunction with other criteria, an adequate process for the selection, acquisition and incorporation of technology in a holistic perspective, so it provides feasible solutions the chemical industry in pursuit of their goals. Then the criterion becomes a benchmark for assessing an appropriate technology transfer process. We performed a theoretical analysis of the technological and environmental criteria, proposing thirty-six (36) technological-environmental criteria interrelated under a systemic approach in the process of transfer of chemical technology, focused on a methodological cycle first run, based primarily on the research-action method. Future research is expected to make a refinement of the criteria from the formulation and validation of metrics so that necessary adjustments are made to optimize the process of transfer of chemical technology.
\end{abstract}

\section{Keywords:}

- technological transference

- environmental criteria

- chemical technology

- holistic focus

- action research method

\section{Introducción}

Ámbitos generales

La transferencia de la tecnología química en las organizaciones y países del mundo es un tema de vital importancia en la actualidad, entendiéndose como un proceso que va desde la selección, hasta la asimilación y difusión en la industria. Los países subdesarrollados donde se evidencia el rezago en ciencia y tecnología se insertan en el proceso de transferencia tecnológica visto en principio como la simple adquisición de la tecnología a un proveedor externo.

Adicionalmente se tiene que el escenario químico internacional está actualmente dominado por grandes empresas, diversificadas e integradas, que conviven con empresas muy especializadas de menor tamaño. Europa, Estados Unidos y Japón, concentran 85\% del mercado y reciben los principales flujos de inversiones directas de la industria (Antunes et al., 2001; DuránGarcía, 2007 y Durán-García et al., 2011).

En la industria química se observan, en los años recientes, innovaciones en los procesos de producción de los sistemas de control, de la instrumentación utilizada, del carácter no contaminante de las tecnologías, tecnologías limpias, etcétera. Las innovaciones de los procesos tienen como objetivo principal mejorar las rutas de producción en busca del aumento de la calidad y competitividad de los productos, respetando las regulaciones y normas, entre ellas las ambientales.
Se puede verificar, a su vez, una tendencia al desarrollo de una "química de funciones" que tiene como objetivo modificar las características de los productos en función de posibles nuevos usos. De la misma forma, como resultado de las presiones ecológicas, se observa una preocupación por la necesidad de disminuir la cantidad y mejorar la calidad de los efluentes industriales, aprovechar los recursos, y reducir el consumo de recursos energéticos y recursos naturales (Antunes et al., 2001; Durán-García, 2007; Durán-García et al., 2011).

En el transcurso de los últimos años, las organizaciones se han encontrado con el inconveniente de que, transferir y adoptar una tecnología, no implica solamente la adquisición, sino también involucra un adecuado proceso de asimilación. Aunado a esto, por lo menos en la fase de la transferencia de tecnología, los países se han encontrado con serios inconvenientes entre los cuales, se mencionan algunos (Durán-García, 2007; Álvarez et al., 2008, Durán-García et al., 2011).

- Cláusulas restrictivas en los convenios de transferencia, como los sistemas de patentes

- Adquisición de tecnologías obsoletas y costosas

- Adquisición de tecnologías que no se adaptan al contexto de la organización receptora

- Problemas con las regulaciones ambientales. Adaptación a las normas de calidad ambiental

Por ejemplo, en la industria química de Brasil y Venezuela se observa el manejo de patentes como estrategia 
tecnológica. Entiéndase por patente el privilegio temporal que el estado concede a una persona -física o moral- por la creación de algo nuevo, con aplicación industrial y que resulta en beneficio para la sociedad (Durán-García, 2007; Durán-García et al., 2011).

En un estudio realizado por estos autores se observa que en Venezuela y Brasil, la mayor parte de las patentes concedidas en Estados Unidos, se refieren a invenciones sometidas al análisis de la USPTO (USPatent\&Trademark Office) las cuales fueron debidamente reconocidas. Una de las conclusiones más importantes de este trabajo es que las empresas estatales de Venezuela y Brasil son las mayores detentoras de patentes, donde INTEVEP concentra $77 \%$ de las patentes en las industrias químicas venezolanas y PETROBRAS tiene 30\% (Durán-García, 2007; Durán-García et al., 2011).

Esto constituye un criterio de importancia para las organizaciones que deseen insertarse en el proceso de adopción de la tecnología química, pues se necesita una adecuada capacidad de negociación con organizaciones de estados que poseen la mayoría de las patentes y/o licencias.

En opinión de Durán-García (2007), Álvarez et al. (2008) y Durán-García et al. (2011), los inconvenientes más comunes en la transferencia de tecnología, son los siguientes:

- Desconocimiento de los diferentes tipos de tecnologías al momento de seleccionar, negociar y adquirir una tecnología.

- Ausencia de un estudio previo que permita por lo menos la selección y adquisición adecuada de una tecnología.

- Obligación por parte de la organización adquirente, de mantener en secreto el conocimiento que se transfiere, más allá de la vigencia del contrato de comercialización de tecnología.

- Legislación aplicable y tribunales competentes para la organización que adquiere la tecnología, en virtud de las posibles diferencias legales que se produzcan. Los aspectos legales deben resolverse según las leyes del país de la organización que cede tecnología.

Este esquema de transferencia lejos de impulsar el desarrollo integral entre ambas partes, propicia una dependencia perjudicial y condiciones antieconómicas, principalmente para la parte que adquiere la tecnología. Este tipo de situación refuerza la marcada diferencia entre organizaciones poderosas y débiles, países desarrollados y subdesarrollados. El escaso balance positivo para la parte del adquirente se traduce, en térmi- nos generales, en un proceso de transferencia con unos escasos matices de asesoría técnica y una negociación más o menos favorable a pesar de las condiciones restrictivas.

Para que la transferencia de tecnología se transforme en un factor de desarrollo tecnológico, se debe tomar conciencia de la importancia de la actividad tecnológica, como factor de progreso en todos los sectores de la comunidad y que, para lograr tal motivación, es necesario establecer una política educacional, que en todos los niveles, promueva el aprendizaje de actividades técnicas.

Esta visión proporciona un contexto más amplio, en cuanto a las implicaciones de la transferencia de tecnología en el desarrollo tecnológico, en virtud de que el desarrollo tecnológico viene impulsado por la educación tecnológica que tengan, no solo los miembros de una organización, sino toda la comunidad de un país. Una vez que se toma clara conciencia del problema de desarrollo tecnológico, se evidencia la estrecha relación entre la transferencia de tecnología y la generación de tecnologías, en donde nociones como compra, transferencia, adecuación, difusión, implantación o generación de tecnologías, son solo formas diferentes en que una organización trata de usar la tecnología para llevar a cabo sus planes de desarrollo.

\section{La transferencia de la tecnología}

Bajo las premisas que se han descrito, Durán-García (2007) presenta en la figura 1 el proceso de transferencia de tecnología, destacando los eventos más importantes y su interrelación.

El primer paso que se menciona en la figura 1 es la detección de necesidades donde se formulan los objetivos específicos, como punto de partida en este complejo proceso de decisión. Para formular los objetivos se hace un análisis de capacidades tecnológicas, de planeación estratégica, tecnológico de la competencia y pronósticos tecnológicos. Esto lleva a la búsqueda de soluciones a través de las alternativas tecnológicas existentes, las cuales deben analizarse comparativamente con las características de las mismas, con el propósito de seleccionar aquellas alternativas que se ajusten a los objetivos que la organización se esté planteando.

Una vez elegida la tecnología se inicia el proceso de negociación, adquisición, asimilación y adaptación. Este proceso se realiza con su respectivo seguimiento y control, de manera que se pueda mejorar en caso de ser necesario. Como se observa en la figura 1, el proceso de búsqueda y análisis de alternativas tecnológicas, que culmina con la selección de la tecnología deseada, de- 


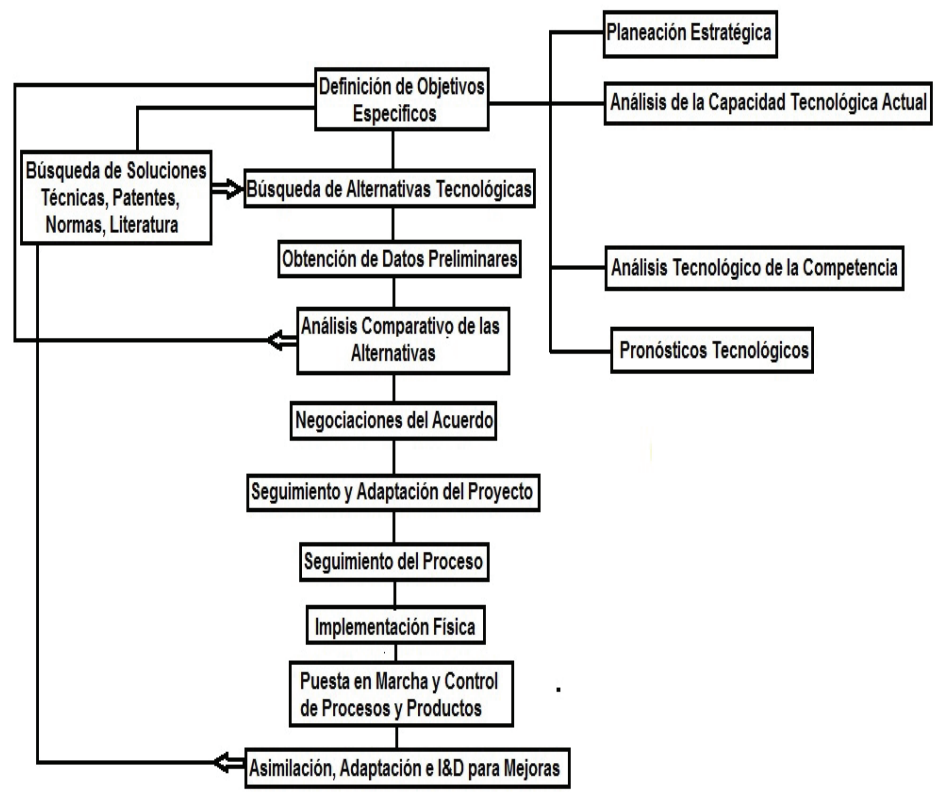

Figura 1. Proceso de transferencia de tecnología desde el enfoque del adquiriente. Fuente: Durán-García (2007) termina en buena parte el éxito de la adopción completa. Entonces, saber de la tecnología apropiada es una de las tareas más complejas de la transferencia de tecnología.

En países en desarrollo, los criterios de selección tecnológica difieren de los aplicados por países industrializados, principalmente por la carencia de recursos para el desarrollo de tecnologías propias. Así, en países en desarrollo se hace hincapié en la selección de la tecnología más adecuada (variando desde la más primitiva a la más avanzada) y su transferencia al país mediante los mejores medios posibles, con el fin de alcanzar el desarrollo económico y social más rápido (Sharif y Sundbajarán, 1983).

En la industria de tratamiento de agua, la selección de la tecnología debe estar enmarcada en el concepto de sostenibilidad, como respuesta a las deficiencias en las tecnologías existentes de los sistemas de tratamiento de agua. Entendiéndose por un sistema sostenible aquel que suministra un servicio con criterio de calidad y eficiencia económica y ambiental, el cual puede ser financiado o cofinanciado por sus usuarios (Galvis y Vargas, 1998). Se evidencia de tal forma que el proceso de selección y transferencia de la tecnología química adecuado, involucra las dimensiones de ambiente, tecnología y comunidad, las cuales se interrelacionan en el contexto político, socioeconómico, cultural, ambiental e institucional. Todo esto hace que la transferencia de la tecnología química sea un proceso de toma de decisiones complejo.

Por otro lado, Antunes et al. (2001) y Durán-García (2007), mencionan que la industria química en América Latina es altamente dependiente de tecnología exóge- na, donde la tecnología que siempre fue considerada un factor importante para el desarrollo económico, se transformó ahora en un elemento estratégico para la creación de ventajas competitivas. Por lo tanto, los países deben llegar a un equilibrio, entre insertarse en el proceso de adopción de la tecnología y un esfuerzo tecnológico endógeno, como estrategia tecnológica que logre los objetivos que se plantean.

En opinión de Durán-García (2007), existe consenso respecto al hecho de que la selección de tecnología en una organización es un proceso de toma de decisiones multifuncional. En la tabla 1, se muestran los criterios manejados por diferentes autores al respecto. Se puede observar en la literatura que la mayoría de las metodologías aplicadas para la selección de tecnologías se basan principalmente en análisis financieros, cuyo parámetro principal es el retorno sobre la inversión. Sin embargo, no puede soslayarse el hecho de que el análisis de aspectos no financieros está ganando un lugar importante en la evaluación de una tecnología al momento de seleccionarse.

Esto se evidencia en la evolución de los criterios presentados, donde en principio se menciona que presenta criterios económicos y de mercado, aunados a criterios de procesos y proyectos, que a su vez, los enfoca en función del ámbito económico-financiero; sin embargo, los criterios de calidad se hacen presentes al momento de seleccionar una tecnología. El criterio de calidad es importante al momento de compararlo con los criterios económicos-financieros, debido a que hay que analizar muy bien cuál es la utilidad y el valor agregado que genera una tecnología de calidad a la organización en 
comparación con la inversión económica que se hace al respecto (Turton, 1998 y Estupiñán et al., 2006).

Por otro lado, clasifica los criterios de selección en económicos, técnicos y sociales, agregando de tal manera valor a los criterios de selección con la inserción de estos dos últimos criterios. Los criterios técnicos toman en cuenta aspectos como dominio de la tecnología, estímulo para la investigación y desarrollo propio de la organización, a partir del posible impacto de la tecnología que se selecciona, cumplimiento de los requisitos de calidad, manejo racional de las materias primas y tecnologías, etcétera. De esta manera, el ámbito técnico permite escudriñar con más detalle las características intrínsecas de las tecnologías que se relacionarán con las demás variables que hay que tomar en cuenta en el proceso de selección de la tecnología.

Entre los criterios sociales se encuentran la creación de empleos que se pueda generar, la contribución de la tecnología a la descentralización industrial, la conservación de valores socioculturales, los efectos de la aplicación de la tecnología en el medio ambiente, etcétera. Por lo tanto, los criterios sociales constituyen una dimensión que involucra el ámbito humano, que se debe tomar en cuenta al momento de analizar la influencia de determinada tecnología en la organización.

Entre los criterios de valores hay que tomar en cuenta el impacto que tiene la selección de una tecnología en la salud y seguridad personal de los miembros de la organización, ambiente, valores, las habilidades personales, el bienestar en general de la organización y su entorno (Sharif y Sundbajarán, 1983).

Este criterio resalta la importancia de cómo una tecnología influye en la idiosincrasia de la organización o país. Además se define claramente cómo un grupo de criterios, los ambientales, se centra en el nivel de contaminación ambiental que se pueda generar y el impacto

Tabla 1. Criterios de selección de tecnología manejados por diferentes autores. Fuente: (Durán, 2007)

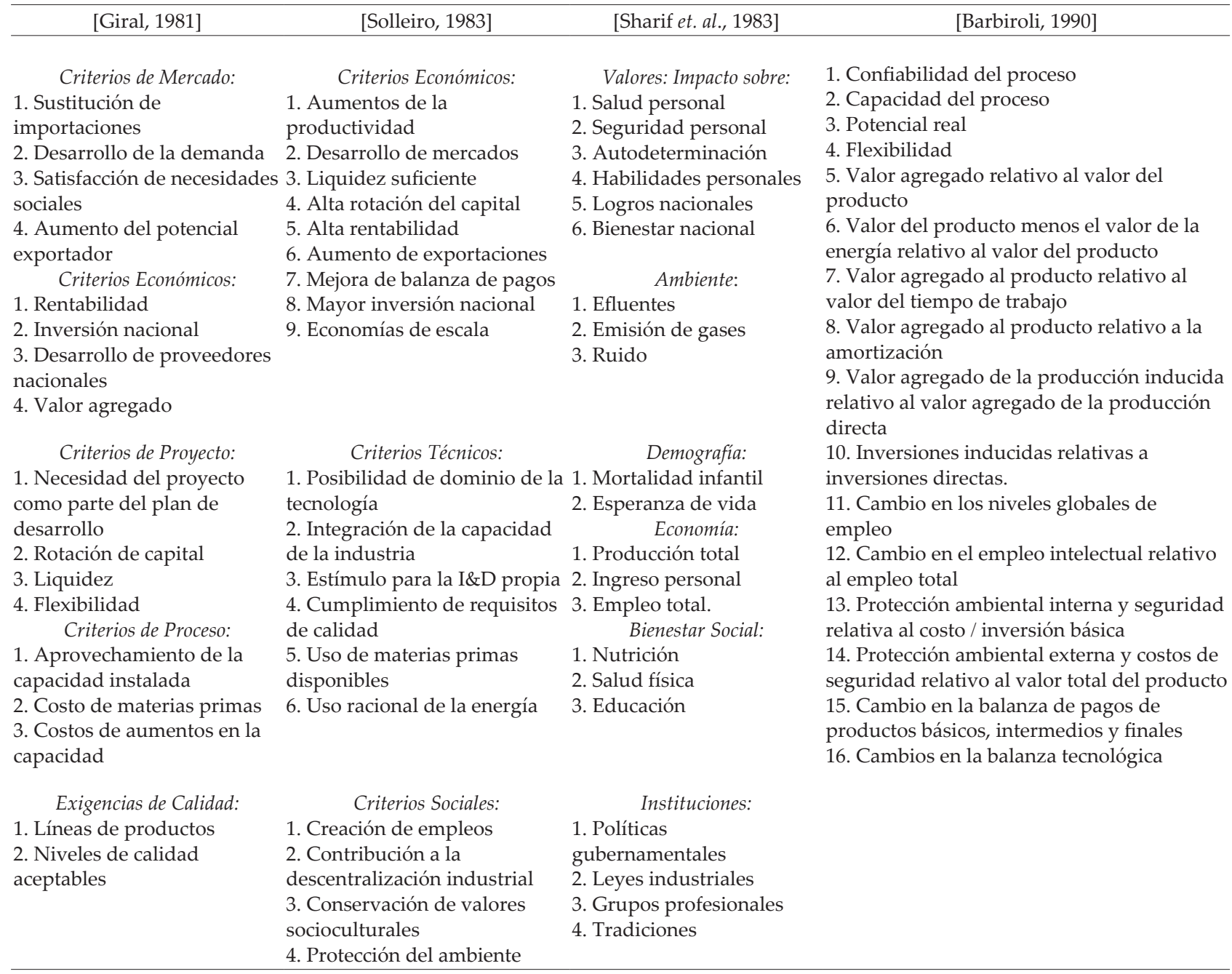


que produce en la organización y su entorno. Por otro lado, los criterios institucionales, entre los cuales se encuentran las políticas gubernamentales, leyes industriales, etcétera, ayudan a enmarcar a la selección de la tecnología dentro de la reglamentación vigente que existe en una organización o país.

Dentro de los criterios propuestos por Sharif y Sundbajarán (1983) no dejan de ser importantes los criterios de demografía y bienestar social. Los criterios de demografía toman en cuenta la influencia de la tecnología seleccionada en la esperanza de vida en general. Estos criterios toman en cuenta el impacto que puede tener una tecnología en la calidad de vida de las personas, constituyéndose en criterios que evalúan aspectos sociales como la ética que hay dentro de la organización y en un país en general.

Finalmente, los criterios resumidos no son discriminados en diferentes ámbitos, tal como lo estructuran los otros autores. Sin embargo, conservan los criterios económicos, sociales, culturales, técnicos y humanos, dándoles un enfoque en función del valor agregado que aporta la selección de la tecnología al cumplir los criterios de selección. El proceso de selección de la tecnología química enmarcado dentro de la adopción de la tecnología química, constituye un proceso de decisión complejo donde hay que tomar en cuenta diversos criterios que permitan lograr con éxito la inserción adecuada de una tecnología en un contexto dado. Se entiende entonces, que el proceso de selección de tecnologías constituye un cuello de botella en el proceso de adopción tecnológica.

Según Landau y Rosemberg (1998), las tecnologías químicas cambian con el tiempo y varían de acuerdo con el campo de conocimiento o sector industrial y las diferencias entre un país y otro, en relación con las tecnologías químicas, dependen tanto de la historia y la cultura de cada país, como del momento en que se incorporaron estas industrias al proceso de industrialización.

Esta idea la complementan Durán-García (2007), Álvarez et al. (2008) y Durán-García et al. (2011), quienes aseguran que las transformaciones ocurridas en los procesos y productos químicos en los últimos años son de tal magnitud, que pueden hacer desaparecer grupos enteros de industrias químicas, por considerarse obsoletas y de costos no competitivos. Debe manejarse este tipo de situaciones a través de un análisis serio que conlleve a la toma de decisiones adecuada en el proceso de transferencia de la tecnología.

Se observa entonces que en el proceso de transferencia de la tecnología química debemos establecer criterios que permitan seleccionar adecuadamente la tecnología. Los criterios incluyen desde los económi- cos, financieros, de mercado, de proyecto, de calidad, de proceso, hasta criterios de tipo social, demográfico, ambiental, institucional y de valores, etcétera. Esto involucra la interacción de aspectos de varias ramas como: ciencias sociales (economía, psicología, sociología, gerencia, etcétera), ciencias exactas (física, química, biología, matemáticas), ciencias aplicadas, donde los criterios y criterios ambientales se encuentran transversalmente a lo largo de todo el proceso de transferencia de la tecnología química.

Por lo tanto, existe una tendencia hacia la integración de las ciencias orientada por la teoría general de los sistemas (Bertalanfy, 1986). Cuando se desarrollan principios de unificación que van más allá de los universos particulares de ciencias diversas, la teoría general de los sistemas se aproxima a los objetivos de la unidad de la ciencia. En el proceso de transferencia tecnológica se observa que existen interrelaciones complejas en un todo indivisible entre los criterios de selección de la tecnología y que no se trata de la suma de criterios particulares que no tienen relación y efectos unos sobre los otros.

Otro aspecto importante es el carácter sistémico que tienen las organizaciones químicas en cuanto a la adquisición de tecnologías, ya que la adquisición de un producto (visto como una nueva tecnología) puede formar parte de un proceso y ambos pueden conformar la tecnología que ha sido transferida y adoptada.

Todo esto lleva a pensar en la siguiente interrogante: ¿Por qué es necesario formular criterios tecnológico-ambientales bajo un enfoque sistémico en la transferencia de la tecnología química? La razón principal está en la dinámica actual de la forma de adquirir tecnologías en las organizaciones. No es sorpresa observar cómo las empresas adquieren tecnología solo por la sencilla razón de que funciona exitosamente en otra organización, porque es una tecnología "novedosa", porque es necesaria en la empresa, y diversas razones, que dejan en evidencia la ausencia de criterios claros que permitan definir si es apropiado o no determinada adopción de tecnología.

La industria química no se escapa de la dinámica cambiante que existe en las organizaciones. Cada día surge la necesidad de mejorar procesos dentro de la industria, lo que hace necesario estudiar la repercusión que tienen los cambios de tecnología química, ya sea por generación propia de la industria o por vía de adopción, en su contexto. A su vez, por la razón expuesta por Antunes et al. (2001) y Durán-García (2007), donde mencionaron que los cambios son de tal magnitud, que pueden llegar a ser grupos enteros de empresas químicas por diversas razones como obsolescencia, costos no competitivos, efectos negativos sobre el ambiente, etcétera. 
Por otro lado, no sólo existe la necesidad de tener en la industria química la tecnología que propicie la satisfacción de sus objetivos, de manera tal que tanto la organización como su medio circundante obtengan beneficios. Existe también la necesidad de cómo lograr dicho objetivo adecuadamente y en el caso de la transferencia de la tecnología química esto es importante. Según Antunes et al. (2001) y Durán-García (2007), una de las principales causas de la desaparición de empresas químicas son los continuos errores que se cometen al adquirir una tecnología con ausencia de criterios de selección. Existen plantas químicas que han tenido cuantiosas pérdidas económicas (calculadas hasta en millones de dólares) o que han ocasionado daños considerables al ambiente por el simple hecho de adquirir una tecnología sin evaluar sus posibles efectos en el contexto de la industria.

Como respuesta a ello se hace necesario proponer criterios que soporten el proceso de transferencia de la tecnología química, de manera que garantice éxito o no en la industria. Una de las características que deben poseer estos criterios es el carácter sistémico, de manera que sean adaptables y tomen en cuenta las complejas interrelaciones que existen entre las variables que inter- vienen en las políticas de decisión tecnológica en una organización como una respuesta a la necesidad actual de transferir tecnología adecuadamente en las industrias químicas.

En virtud de la complejidad que representa el proceso de transferencia de la tecnología química, es fundamental establecer un conjunto de criterios que permitan entender las interrelaciones entre las variables que engloban a este proceso para hacer un mejor análisis del mismo y poder brindar apoyo a este tipo de decisión.

\section{Marco metodológico}

Se proponen las actividades que deben llevarse a cabo, con el fin de alcanzar el objetivo planteado, a través de un marco metodológico (Pérez et al., 2004) el cual se basa principalmente en el método de investigación-acción. Se abordan los primeros siete pasos del ciclo metodológico que originan como producto los criterios tecnológicoambientales en la transferencia de la tecnología química, y que servirán para la elaboración de las respectivas mé-

\begin{tabular}{|c|c|}
\hline Actividad & Descripción \\
\hline $\begin{array}{l}\text { 1. Investigación } \\
\text { documental y bibliográfica }\end{array}$ & $\begin{array}{l}\text { Etapa de la fase Diagnosticar, la cual consta de la revisión } \\
\text { bibliográfica, de los criterios tecnológico-ambientales del proceso } \\
\text { de transferencia tecnológica en la industria química }\end{array}$ \\
\hline $\begin{array}{l}\text { 2. Análisis de los criterios } \\
\text { existentes }\end{array}$ & $\begin{array}{l}\text { Etapa de la fase Diagnosticar. Se analizan los criterios existentes } \\
\text { con el fin de identificar y definir los criterios industriales y } \\
\text { comunes en la transferencia de la tecnología química }\end{array}$ \\
\hline $\begin{array}{l}\text { 3. Formulación de los } \\
\text { objetivos y alcance de la } \\
\text { investigación }\end{array}$ & $\begin{array}{l}\text { Etapa de la fase Planificar la acción. En esta etapa se definen } \\
\text { claramente los objetivos de la investigación, así como las } \\
\text { directrices y espacio de trabajo de los criterios en la transferencia } \\
\text { de la tecnología química }\end{array}$ \\
\hline $\begin{array}{l}\text { 4. Formulación de } \\
\text { la metodología de } \\
\text { investigación }\end{array}$ & $\begin{array}{l}\text { Etapa de la fase de Planificar la acción. En ella se realiza una } \\
\text { adaptación del método investigación-acción con la metodología } \\
\text { DESMET al contexto de la presente investigación. El objetivo } \\
\text { es elaborar el marco metodológico que soporta el trabajo de } \\
\text { investigación }\end{array}$ \\
\hline $\begin{array}{l}\text { 5. Formulación de los } \\
\text { criterios industriales y } \\
\text { termodinámicos }\end{array}$ & $\begin{array}{l}\text { Etapa de la fase Tomar la acción. En esta etapa se toman en cuenta } \\
\text { las posibles características y matices encontrados en los criterios } \\
\text { existentes y la revisión bibliográfica para definir claramente } \\
\text { los criterios tecnológico-ambientales en la transferencia de la } \\
\text { tecnología química en una versión de prueba }\end{array}$ \\
\hline 6. Análisis de contexto & $\begin{array}{l}\text { Etapa de la fase Tomar la acción. Se determinan las } \\
\text { especificaciones y acuerdos necesarios para implementar la } \\
\text { evaluación de los criterios propuestos. El objetivo es seleccionar el } \\
\text { método de evaluación y el análisis del contexto de evaluación para } \\
\text { aplicar DESMET }\end{array}$ \\
\hline $\begin{array}{l}\text { 7. Conclusiones y } \\
\text { recomendaciones }\end{array}$ & $\begin{array}{l}\text { Etapa de la fase Especificar el aprendizaje. Se establecen algunas } \\
\text { conclusiones relativas a los criterios definidos y resultados } \\
\text { preliminares }\end{array}$ \\
\hline
\end{tabular}

Tabla 2. Fases de la metodología investigación-acción propuesta. Fuente: Adaptado Durán et al. (2011) 
tricas que cuantificarán en una futura investigación la pertinencia y adecuación de las mismas.

Estos criterios están enmarcados dentro del Modelo sistémico para la adopción de la tecnología por la Industria Química propuesto por Durán-García et al. (2011), el cual ubica ámbitos sociales, técnicos, ambientales, industriales, culturales, organizacionales, científicos que intervienen en el proceso de transferencia tecnológica. Este ciclo propuesto puede repetirse $n$ veces, pero para efectos de esta investigación se considera una primera iteración que se puede observar en la tabla 2.

\section{Resultados y discusión}

Los modelos analizados y las prácticas en la ingeniería (conocimiento adquirido con la experiencia por expertos en el manejo de la adopción tecnológica, prácticas básicas, etcétera) ofrecen una variada y amplia cantidad de criterios que se utilizan a conveniencia para la selección, adquisición, transferencia y adopción de la tecnología, respectivamente.

Por ejemplo, Durán-García (2007), en el manual de contratación de tecnología del CINDA, describen algunos aspectos y criterios a tomar en cuenta en la contratación y negociación tecnológica como eje fundamental de la transferencia: inversión extranjera, asistencia técnica, contratación de expertos, contratación de licencia y patentes, la disponibilidad de la información, poder de negociación, las características intrínsecas y técnicas de la tecnología, etcétera.

Por otro lado, Álvarez et al., (2008) proponen que existen algunas variables que intervienen en la asimilación de la tecnología en relación con su respectivo contexto, entre los cuales se encuentran: relaciones entre organizaciones similares, impacto de las políticas y procedimientos del estado, efecto de la asimilación en la estructura de precios de bienes que ofrece la organización, asistencia técnica, relación de la organización con el entorno socio-cultural, y otros.

En cuanto a la tecnología química, la adopción presenta algunos criterios y características esenciales a tomar en cuenta. Turton (1998), Antunes et al. (2001), Rivera (2002), Álvarez et al. (2005), Mendoza et al. (2005), Rincón et al. (2005), Estupiñan et al. (2006), Rincón (2006), Durán-García (2007), Álvarez et al. (2008) y Durán-García et al. (2011), proponen que antes de realizar la adquisición de una tecnología química en particular, se debe realizar un estudio de factibilidad que permita incluir factores o criterios como: calidad de la tecnología, costo de la misma, inversión en maquinarias y equipos, suministro de materias primas, acceso a mercados internacionales, regulaciones ambientales, nor- mativas legales, bases de diseño del proceso químico, prácticas bases en los procesos industriales, especificaciones de productos, materias primas e insumos, manejo de efluentes, desechos y subproductos no deseados, etcétera.

A su vez, consideran que la adquisición de la tecnología en el campo químico y petroquímico requiere que se estudien los siguientes aspectos: en los procesos continuos la ingeniería básica, ingeniería de detalle, catalizador, descripción de las características del proceso y mejoras futuras. En el caso de procesos por lote se requiere especificar la formulación (fórmula del producto), procedimiento de producción, aplicación, ingeniería y operación de la planta y mejoras futuras. Para ambos procesos se requiere la búsqueda de la organización licenciante o proveedor de la tecnología bajo las condiciones más beneficiosas para las partes involucradas.

Un criterio o aspecto preponderante en la adopción de la tecnología química es el factor ambiental asociado a la tecnología que se quiere asimilar. En este sentido, Turton (1998), Antunes et al. (2001), Rivera (2002), Álvarez et al. (2005), Rincón et al. (2005), Álvarez et al. (2008) y Durán-García et al. (2011) describen la realidad asociada a la tecnología química adoptada por organizaciones de países subdesarrollados como Venezuela. A pesar de poseer una política ambiental de Estado recién creada y establecida en los últimos 40 años, se han evidenciado realidades donde hay un efecto contaminante por parte de una tecnología química adoptada.

Este es el caso de los primeros desarrollos del Complejo Petroquímico de Morón, el cual generó un importante impacto ambiental, pues al instalarse las primeras plantas, las tecnologías disponibles presentaban alto poder contaminante, específicamente la producción de cloro soda, y no existían dispositivos para el control de las descargas, las cuales durante mucho tiempo afectaron de manera significativa los medios circundantes.

Sin embargo, describen que en países subdesarrollados con normativas ambientales legales como Venezuela han existido experiencias de montaje de plantas de tratamiento de efluentes que han minimizado los daños al medio ambiente a pesar de la incorporación de nuevas tecnologías. La primera planta de tratamiento de efluentes fue incorporada en el Complejo Petroquímico El Tablazo, la cual pasó por un proceso gradual de adaptación hasta llegar a cumplir su objetivo de minimizar los efectos contaminantes.

La ausencia de modelos de transferencia de la tecnología química no es una limitante en la recolección de los criterios usados comúnmente en la práctica de ingeniería, los cuales son necesarios para realizar la adopción apropiada. En consecuencia, se presentan treinta y 
seis criterios (36) que han sido conceptualizados como producto de un análisis de los modelos y las investigaciones descritas.

1. Mano de obra. Se refiere a los recursos humanos disponibles que se encargan de operar la planta química.

2. Calidad y requerimientos de insumos. Disponibilidad y capacidad técnica de procesamiento.

3. Impacto ecológico. Asociado a los efectos o consecuencias que se generan en el medio ambiente por la asimilación y uso de la tecnología.

4. Calidad del producto. Nivel de satisfacción generado en el consumidor del producto.

5. Patrón de consumo. Se refiere al impacto que genera la tecnología en el consumo de la población que se beneficia de la misma.

6. Costo de la tecnología. Relacionado al valor monetario que acarrea la adquisición, transferencia y asimilación de la tecnología química.

7. Duración del proyecto. Referido a la velocidad con que se puntualiza la adquisición, transferencia y asimilación de la tecnología. Esto sugiere la utilización de estrategias como la persuasión, satisfacción de intereses mínimos y conocimiento del beneficio que se genera al realizar la negociación por ambas partes.

8. Capacidad de asimilación. Asociado con el nivel de aprovechamiento de la tecnología por parte de la organización en función de sus capacidades tecnológicas.

9. Perfil del personal. Referido a las características mínimas de los profesionales, técnicos y obreros involucrados directamente con la tecnología, que satisfagan las necesidades de la planta química.

10. Generación de productos y subproductos. Productos que se obtienen como consecuencia de la tecnología adoptada por la planta química o a partir de la planta química adoptada.

11. Generación de desechos. Cantidad de desechos que se generan una vez adoptada la tecnología química por la planta.

12. Vida útil de la planta. Asociado al tiempo útil de funcionamiento de la planta que se adopta o la planta donde se va incorporar una nueva tecnología.

13. Condiciones de operación. Se refiere al nivel de severidad de operación de la planta química que se quiere adoptar o las limitaciones de operación al incorporar una tecnología en la planta química.

14. Límites de expansión. Restricciones a considerar en la planta química en cuanto a su capacidad para expandirse.

15. Nivel de producción. Efecto generado en el nivel de producción de la organización sin afectar significativamente sus objetivos y razón de ser.
16. Origen del proveedor. Referido a la identidad del proveedor, experiencias previas en transferencias tecnológicas, esquemas de negociación utilizados, intereses de la organización proveedora, capacidad para licenciar, normas que utiliza con frecuencia, etcétera.

17. Manejo de la información. Se refiere a la información que proporcionan los antecedentes de la tecnología en otros contextos, la descripción de tecnologías similares y comparables, tecnologías alternativas y existentes que se puedan adoptar. Esta información promueve la selección de la tecnología química apropiada.

18. Clasificación de la tecnología. Referido al tipo de tecnología a seleccionar: básica, obsoleta, avanzada, de punta, etcétera. Esto requiere el conocimiento previo de antecedentes que explican por qué una tecnología se clasifica de una manera particular.

19. Madurez de la tecnología. Asociado con el punto donde se encuentra la tecnología que se quiere adoptar en su ciclo de vida: tecnologías en crecimiento, desarrollo, maduras o tecnologías en degradación.

20. Ventajas en el producto. Asociado con las ventajas competitivas de la tecnología para el comprador. La organización opta por una tecnología debido a sus condiciones atractivas, dándole prioridad al beneficio global que se genera.

21. Requerimientos energéticos. Son las necesidades de energía (eléctrica, eólica, térmica, etcétera) que requiere la planta química.

22. Patentes y licencias. Se refiere al grado de protección que tiene una tecnología por parte del proveedor. Generalmente se relaciona con el conocimiento asociado con la tecnología, el cual se encuentra privilegiado por una licencia o patente.

23. Requerimientos de almacenaje. Necesidades de niveles de almacenaje de los insumos y productos que se generan en la planta química.

24. Adaptabilidad. Capacidad de aplicar la tecnología adoptada en diferentes contextos de aplicación de plantas químicas.

25. Canal de transferencia. Se refiere a los actores y procesos que participan en el modelo. Suele considerarse en estos modelos la participación de la dupla proveedor-comprador, pero también se tiene la presencia de terceros o intermediarios. A su vez, el modelo puede estar enmarcado en inversiones extranjeras, alianzas entre empresas y corporaciones, etcétera.

26. Factor ambiental. Se refiere a las políticas ambientales que manejan las organizaciones involucradas con el fin de minimizar los efectos perjudiciales en el ambiente. Requiere disponibilidad y voluntad para 
instalar plantas de tratamiento, crear programas de disminución de contaminantes y reciclaje, etcétera.

27. Asistencia técnica. Capacidad de ayuda especializada que provee el que vende la tecnología, garantizando el mejor entendimiento de la asimilación de la tecnología en la realidad y entorno de la organización adquirente.

28. Contratación de expertos. Asociado con la adquisición del personal calificado y competente que garantice la adecuada manipulación de la tecnología en el entorno donde se adopta. Puede ser complemento de la asistencia técnica que proporciona el proveedor.

29. Desechos y residuos contaminantes. Contaminantes que se generan como subproductos de los procesos asociados con la planta química que perjudican el medio ambiente.

30. Grado de automatización. Nivel de automatización de los procesos (operaciones unitarias) que se realizan en la planta.

31. Riesgos. Capacidad de asumir las consecuencias de las decisiones tomadas en torno a la adopción: riesgos económicos, riesgos sanitarios, riesgos ambientales, etcétera.

32. Historia de la tecnología. Asociada con el desempeño previo de la tecnología en distintos escenarios. Son los antecedentes de la tecnología al ser aplicada en otros contextos.

33. Impacto en los procesos. Se refiere a los efectos que se producen en los procesos asociados (operaciones unitarias): mejoras, cambios parciales en los mismos, reestructuraciones, eliminación de subprocesos, etcétera.

34. Descripción de la tecnología. Se refiere a la descripción de los procesos, diagramas de flujo que indican equipos utilizados, balance de materiales y energía, espacio físico a utilizar, condiciones de presión y temperatura, ingeniería básica, ingeniería de detalle, ingeniería de la planta.

35. Impacto en las unidades operativas. Efectos producidos en las unidades de producción de la organización: eliminación de maquinarias o equipos, recarga en la operación de los equipos existentes e inserción de nuevos equipos en los procesos de operaciones unitarias (procesos de separación).

36. Criterios técnicos propios del caso. Asociados con las características técnicas y particulares de las plantas químicas con sus procesos inherentes.

Por lo tanto, en la transferencia de la tecnología química hay que establecer criterios entre los cuales se encuentran transversalmente los tecnológico-ambientales bajo un enfoque sistémico, cuyos insumos son la combi- nación de aspectos de modelos existentes, criterios en trabajos previos y las "prácticas básicas" en ingeniería que permitan transferir adecuadamente la tecnología.

\section{Procedimiento de evaluación de los criterios tecnológico-ambientales}

Estos criterios se proponen en una primera versión dentro del ciclo metodológico, por lo que una vez que se evalúan a través de sus métricas de acuerdo con el método de evaluación de la metodología DESMET; se realizará la refinación de los mismos de forma que se adapten lo mejor posible al proceso sistémico de transferencia de la tecnología química (Turton et al., 1998).

DESMET es una metodología que permite identificar claramente el contexto existente en la evaluación de los criterios tecnológico-ambientales, así como las limitaciones y puntos fuertes de los mismos, a través de condiciones favorables presentes y no presentes en cada uno de los métodos de evaluación. Esta metodología se compone de nueve técnicas, entre las cuales están; las encuestas cuantitativas, los análisis de características por proyección, por caso de estudio, por experimento y encuestas, entre otros.

De estos se selecciona para la evaluación de los criterios actuales, el método de evaluación denominado: "Análisis de características por encuestas", por integrar la mayor cantidad de condiciones favorables presentes en el mismo. Este método es útil cuando es difícil cuantificar los beneficios y cuando se conoce la población de usuarios que utilizarán los criterios, permitiendo seleccionar apropiadamente los expertos evaluadores, que hayan utilizado estos criterios en proyectos previos.

Una vez seleccionado el método y los evaluadores que participarán, se deben entregar los cuestionarios (en físico o en digital) a cada uno de los expertos para que evalúen los criterios en función de las métricas asociadas a cada uno. Para seleccionar los criterios definitivos, el nivel de aceptación de cada métrica deberá ser de $75 \%$, donde cada respuesta positiva dada por los expertos tendrá el valor de uno (1).

Para cuantificar si una métrica asociada con el criterio es aceptada o no, se deben considerar las cuatro respuestas para cada pregunta a evaluar, en función del nivel de pertinencia, factibilidad, profundidad y escala de las métricas del modelo, como se muestra en la tabla 3. Si una métrica no supera el nivel mínimo de aceptación de $75 \%$ debe desecharse al momento de validar los criterios tecnológico-ambientales que permitirán conocer si una tecnología se ha transferido correctamente. 
Un ejemplo de formulación de las métricas asociadas a algunos de los criterios tecnológico-ambientales se presentan en la tabla 4, la cual es parte del instrumento que se aplicará a los expertos para completar el proceso de validación de los criterios y como consecuencia la refinación de los mismos, para que sean aplicados en un proceso de transferencia de tecnología química ya realizado.

\section{Conclusiones}

Basándose en los resultados obtenidos y el análisis de los mismos, es posible indicar algunas conclusiones:

Se proponen en principio criterios tecnológico-ambientales que han sido conceptualizados, producto de un análisis de los criterios que se derivan de los mode- los e investigaciones previas. Estos criterios en conjunto con las bases conceptuales son insumos para la propuesta de los criterios en la transferencia de la tecnología química.

La formulación de los criterios tecnológico-ambientales en la transferencia de la tecnología química se fundamentó en las más recientes investigaciones realizadas en el ámbito académico (Bertalanffy, 1986; Antunez et al., 2001; Álvarez et al., 2005; Landau y Rosemberg, 1998; Mendoza et al., 2005; Pérez et al., 2004; Rincón, 2006; Rincón et al., 2005; Rivera, 2002; Sharif y Sundbajarán, 1983; generándose como producto la formulación de treinta y seis (36) criterios tecnológico-ambientales a ser evaluados por los expertos, a través de sus respectivas métricas en una futura investigación.

Tabla 3. Características específicas para la evaluación de cada métrica. Fuente: adaptado de Durán et al. (2011)

\begin{tabular}{lll}
\hline \multicolumn{1}{c}{ Característica general } & \multicolumn{1}{c}{ Descripción } & \multicolumn{1}{c}{ Escala } \\
\hline Pertinencia de la métrica & $\begin{array}{l}\text { Se refiere a si la métrica es adecuada para } \\
\text { medir la existencia o no de la característica } \\
\text { donde se encuentra }\end{array}$ & $\begin{array}{l}\text { 1: significa que la métrica es pertinente } \\
\text { 0: significa que la métrica no es pertinente }\end{array}$ \\
Factibilidad de la métrica & $\begin{array}{l}\text { Se refiere a si es factible medir la característica } \\
\text { propuesta en la métrica dentro del contexto de } \\
\text { evaluación }\end{array}$ & $\begin{array}{l}\text { 1: significa que la métrica es factible } \\
\text { Nivel de profundidad que la métrica no es factible }\end{array}$ \\
& $\begin{array}{l}\text { Se refiere a si la métrica a verificar tiene el } \\
\text { nivel de profundidad adecuado para que el } \\
\text { resultado sea relevante }\end{array}$ & $\begin{array}{l}\text { 1: significa que la métrica tiene el nivel de } \\
\text { profundidad adecuado } \\
\text { 0: significa que se requiere una mayor } \\
\text { profundidad en la métrica }\end{array}$ \\
Escala de la métrica & $\begin{array}{l}\text { Se refiere a si la escala propuesta es adecuada } \\
\text { para medir la métrica }\end{array}$ & $\begin{array}{l}\text { 1: significa que la escala es adecuada } \\
\text { 0: significa que la escala no es adecuada }\end{array}$ \\
\hline
\end{tabular}

Tabla 4. Métricas asociadas a los criterios (ejemplo del instrumento de validación). Fuente: adaptado de Durán et al. (2011)

\begin{tabular}{|c|c|c|c|}
\hline Criterio & Métrica & Pregunta & Formulación \\
\hline \multirow[t]{2}{*}{$\begin{array}{l}\text { Compuestos no deseados } \\
\text { (Desechos y residuos } \\
\text { contaminantes, criterio 29) }\end{array}$} & $\begin{array}{l}\text { Información de los } \\
\text { compuestos no deseados }\end{array}$ & $\begin{array}{l}\text { ¿Se documentaron las especificaciones } \\
\text { de los compuestos no deseados que se } \\
\text { generan? }\end{array}$ & $\begin{array}{l}0=\mathrm{No} \\
1=\mathrm{Si}\end{array}$ \\
\hline & $\begin{array}{l}\text { Aprovechamiento de los } \\
\text { compuestos no deseados }\end{array}$ & $\begin{array}{l}\text { ¿Se documentó el aprovechamiento de } \\
\text { los compuestos no deseados? }\end{array}$ & $\begin{array}{l}0=\mathrm{No} \\
1=\mathrm{Si}\end{array}$ \\
\hline \multirow[t]{2}{*}{$\begin{array}{l}\text { Impacto en los procesos } \\
\text { (criterio 33) }\end{array}$} & $\begin{array}{l}\text { Mejoras en los procesos } \\
\text { Cambios y reestructuraciones } \\
\text { parciales en los procesos }\end{array}$ & $\begin{array}{l}\text { ¿Se documentó la evaluación de las } \\
\text { mejoras de los procesos industriales? } \\
\text { ¿Se documentó la evaluación de } \\
\text { la pertinencia en los cambios y } \\
\text { reestructuraciones parciales de los } \\
\text { procesos? }\end{array}$ & $\begin{array}{l}0=\mathrm{No} \\
1=\mathrm{Si} \\
0=\mathrm{No} \\
1=\mathrm{Si}\end{array}$ \\
\hline & Cambios de subprocesos & $\begin{array}{l}\text { ¿Se documentó la evaluación de los } \\
\text { posibles cambios de los subprocesos? }\end{array}$ & $\begin{array}{l}0=\mathrm{No} \\
1=\mathrm{Si}\end{array}$ \\
\hline \multirow{2}{*}{$\begin{array}{l}\text { Requerimientos } \\
\text { energéticos (Energía, } \\
\text { criterio 21) }\end{array}$} & Fuentes de Energía & $\begin{array}{l}\text { ¿Se documentaron las fuentes de energía } \\
\text { requeridas en los procesos industriales? }\end{array}$ & $\begin{array}{l}0=\mathrm{No} \\
1=\mathrm{Si}\end{array}$ \\
\hline & Correspondencia de energía & $\begin{array}{l}\text { ¿Se documentó la correspondencia } \\
\text { entre las diferentes formas de energía } \\
\text { requeridas y las disponibles? }\end{array}$ & $\begin{array}{l}0=\mathrm{No} \\
1=\mathrm{Si}\end{array}$ \\
\hline
\end{tabular}


La aplicación de la metodología investigación-acción permitió, entre otras cosas realizar todos los pasos hasta llegar a la fase previa de la evaluación de los criterios propuestos.

\section{Referencias}

Álvarez M., Rincon G., Pérez M. Initiatives to Face the Logistic Challenges of the Clean Fuel Age: A Software Selection Case, World Congress of Chemical.

Engineering Glasgow, Reino Unido JuPOR (7a, Reino Unido, 2005), CD: Congress Manuscripts 7th World Congress Chemical Engineering, 2005, pp. 1-8.

Álvarez M., Rincón G., Pérez M., et al. Evaluation and Selection of Discrete-Event Simulation Software for the Oil Industry. Latin American Applied Research, volumen 38, 2008: 305-312.

Antunes A., Souza C., Dutra L. Desarrollo de la tecnología en la Industria Química de Brasil y Venezuela: Énfasis en patentes. Revista Espacios, volumen 22 ( número 2), 2001: 1-8.

Bertalanffy V. Nuevos patrones de pensamiento biológico y médico, perspectivas en la teoría general de sistemas, segunda reimpresión, 1986, p. 92.

Durán M. Propuesta de modelo sistémico para la adopción de la tecnología química, (tesis de maestría en ingeniería de sistemas), Venezuela, Universidad Simón Bolívar, 2007, 175 p.

Durán-García M., Pérez M., Rincón G., Mendoza L. Modelo sistémico para la adopción de la tecnología por la industria química. DYNA Ingeniería e Industria, volumen 86 (número 5), noviembre 2011: 581-588.

Estupiñán C., Flórez A., Flórez M. Caracterización mecánica y termodinámica de vidrios no óxidos para materiales fotónicos, Revista de la Sociedad Colombiana de Física, volumen 38 (número 4), 2006: 1387-1390.

Galvis A., Vargas V. Modelo de selección de tecnología en el tratamiento de agua para consumo humano, en: Primer seminario agua y sostenibilidad conferencia internacional, Colombia, Memorias del Congreso, Cali, Colombia, 1998, pp. 1-5.

Landau A., Rosemberg N. Chemicals and Long-Term Economic Growth: Insights from the Chemical Industry, United States of America, John Wiley \& Sons, Inc, 1998, pp. 15-20.
Mendoza L., Pérez M., Grimán A. Propuesta del modelo sistémico de calidad (MOSCA) del Software. Revista Computación y Sistemas, volumen 8 (número 3), 2005: 196-221.

Pérez M., Rojas T., Mendoza L., et al. Systemic Methodological Framework for IS Research, en: 10th Proceedings of the Tenth Americas Conference on Information Systems, New York, 2004, pp.1-15.

Rincón G. Etapas de un proyecto para la industria de procesos (trabajo de ascenso a la categoría de asociado en el área de procesos químicos), Departamento de Procesos y Sistemas, Universidad Simón Bolívar, Caracas Venezuela, 2006, 120 p.

Rincón G., Pérez M., Álvarez M., et al. A Discrete-Event Simulation and Continuous Software Evaluation on a Systemic Quality Model: an Oil Industry Case. Information \& Management, volumen 42 (número 8), 2005: 1051-1061.

Rivera C.A. Análisis termodinámico y planeación operacional de sistemas generadores de potencia, (tesis de maestría en ciencias en ingeniería mecánica), Instituto Politécnico Nacional, México, 2002, pp. 1-137.

Sharif M. y Sundbajarán V. Quantitative Model for the Evaluation of Technological Alternatives. Technological Forecasting and Social Change, 1983, pp. 15-29.

Turton R, Bailie R., Whiting W., et al. Analysis Synthesis and Design of Chemical Processes, 2da edición, Nueva Jersey, EUA, Prentice Hall PTR, 1998, pp.1-350.

\section{Este artículo se cita:}

\section{Citación estilo Chicago}

Durán-García, Martín Enrique. Criterios tecnológico-ambientales bajo un enfoque sistémico: transferencia de tecnología química. Ingeniería Investigación y Tecnología, XV, 03 (2014): 339-350.

\section{Citación estilo ISO 690}

Durán-García M.E. Criterios tecnológico-ambientales bajo un enfoque sistémico: transferencia de tecnología química. Ingeniería Investigación y Tecnología, volumen XV (número 3), julio-septiembre 2014: 339-350.

\section{Semblanza del autor}

Martín Enrique Durán-García. Es ingeniero químico con maestría en ingeniería de sistemas. Obtuvo el doctorado en ingeniería por la Universidad Simon Bolivar. Es profesor en la misma institución y labora en el Departamento de Tecnología Industrial, es coordinador de mantenimiento en ingeniería, evaluador de proyectos de investigación y desarrollo de la Universidad Simón Bolivar. Sus áreas de investigación son: tecnología de transferencia, modelos sistémicos, energía limpia y termodinámica. 